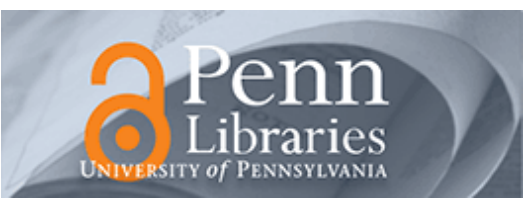

University of Pennsylvania

ScholarlyCommons

2-23-2009

\title{
Rectifying junctions of tin oxide and poly(3-hexylthiophene) nanofibers fabricated via electrospinning
}

\author{
N J. Pinto \\ University of Puerto Rico \\ K V. Carrasquillo \\ University of Puerto Rico \\ Christopher M. Rodd \\ University of Pennsylvania, rodd@seas.upenn.edu \\ Ritesh Agarwal \\ University of Pennsylvania, riteshag@seas.upenn.edu
}

Follow this and additional works at: https://repository.upenn.edu/mse_papers

\section{Recommended Citation}

Pinto, N. J., Carrasquillo, K. V., Rodd, C. M., \& Agarwal, R. (2009). Rectifying junctions of tin oxide and poly(3-hexylthiophene) nanofibers fabricated via electrospinning. Retrieved from

https://repository.upenn.edu/mse_papers/162

Rectifying junctions of tin oxide and poly(3-hexylthiophene) nanofibers fabricated via electrospinning Nicholas J. Pinto, Katherine V. Carrasquillo, Christopher M. Rodd, and Ritesh Agarwal, Appl. Phys. Lett. 94, 083504 (2009), DOI:10.1063/1.3089878

Copyright 2009 American Institute of Physics.

This article may be downloaded for personal use only. Any other use requires prior permission of the author and the American Institute of Physics. Reprinted in Applied Physics Letters, Vol. 94, 083504

Publisher URL: http://link.aip.org/link/?APPLAB/94/083504/1

This paper is posted at ScholarlyCommons. https://repository.upenn.edu/mse_papers/162

For more information, please contact repository@pobox.upenn.edu. 


\title{
Rectifying junctions of tin oxide and poly(3-hexylthiophene) nanofibers fabricated via electrospinning
}

\author{
Abstract \\ Abstract: A fast, simple, and inexpensive method to fabricate in air, $p$-n diodes using electrospun tin oxide \\ nanoribbons and regioregular poly(3-hexylthiophene) nanofibers is described. In addition to being a \\ rectifier under ambient illumination or in the dark, the advantage of our design is the complete exposure \\ of the rectifying nanojunction to the surrounding environment, making them attractive candidates in the \\ potential fabrication of low power consumption diodes and sensors. The diode characteristics were \\ analyzed using the standard diode equation and its use as a UV light sensor was examined.
}

\section{Comments}

Rectifying junctions of tin oxide and poly(3-hexylthiophene) nanofibers fabricated via electrospinning Nicholas J. Pinto, Katherine V. Carrasquillo, Christopher M. Rodd, and Ritesh Agarwal, Appl. Phys. Lett. 94, 083504 (2009), DOI:10.1063/1.3089878

Copyright 2009 American Institute of Physics.

This article may be downloaded for personal use only. Any other use requires prior permission of the author and the American Institute of Physics. Reprinted in Applied Physics Letters, Vol. 94, 083504

Publisher URL: http://link.aip.org/link/?APPLAB/94/083504/1 


\title{
Rectifying junctions of tin oxide and poly(3-hexylthiophene) nanofibers fabricated via electrospinning
}

\author{
Nicholas J. Pinto, ${ }^{1, a)}$ Katherine V. Carrasquillo, ${ }^{1}$ Christopher M. Rodd, ${ }^{2}$ and \\ Ritesh Agarwal ${ }^{2}$ \\ ${ }^{1}$ Department of Physics and Electronics, University of Puerto Rico-Humacao, Humacao, \\ Puerto Rico 00791, USA \\ ${ }^{2}$ Department of Materials Science and Engineering, University of Pennsylvania, Philadelphia, \\ Pennsylvania 19104, USA
}

(Received 10 December 2008; accepted 5 February 2009; published online 25 February 2009)

\begin{abstract}
A fast, simple, and inexpensive method to fabricate in air, $p$ - $n$ diodes using electrospun tin oxide nanoribbons and regioregular poly(3-hexylthiophene) nanofibers is described. In addition to being a rectifier under ambient illumination or in the dark, the advantage of our design is the complete exposure of the rectifying nanojunction to the surrounding environment, making them attractive candidates in the potential fabrication of low power consumption diodes and sensors. The diode characteristics were analyzed using the standard diode equation and its use as a UV light sensor was examined. (C) 2009 American Institute of Physics. [DOI: 10.1063/1.3089878]
\end{abstract}

Most solid state devices incorporate at least one interface between a $p$ - and an $n$-type semiconductor, hence the $p$ - $n$ junction is the focus of much research. The discovery of organic conducting conjugated polymers ${ }^{1}$ made it possible to fabricate reliable devices and sensors in ordinary laboratory conditions. Metal oxides are also desirable materials for use in devices. ${ }^{2}$ Of particular interest is tin oxide $\left(\mathrm{SnO}_{2}\right)$ (Refs. 3 and 4) as it is stable in air, optically transparent, and semiconducting, with a band gap of $\sim 3.4 \mathrm{eV}$. The simplest and easiest hybrid device to fabricate is a diode in which a junction of an organic conducting polymer with an $n$-doped inorganic semiconductor is formed. Such architectures have been typically realized via spin coating ${ }^{5}$ or electrochemical polymerization. ${ }^{6,7}$ Earlier, we reported on the fabrication of hybrid Schottky nanodiodes via electrospinning. ${ }^{8}$ Herein, we report on the same method to fabricate in air and within seconds, $p$ - $n$ diodes, by simply crossing $n$-doped $\mathrm{SnO}_{2}$ and $p$-doped poly(3-hexylthiophene-2-5-diyl) (P3HT) nanofibers. The devices operate well under ambient illumination or in the dark, with on/off ratios of $\sim 25$. Although several studies have been done on $\mathrm{SnO}_{2} / \mathrm{P} 3 \mathrm{HT}$ composites, ${ }^{9-11}$ primarily for use as sensors, this is the first study on $p$ - $n$ diodes fabricated using nanofibers of pure P3HT and nanoribbons of $\mathrm{SnO}_{2}$. The advantage of our design is its quasi-onedimensional feature and the complete exposure of the nanojunction to the surrounding environment making them attractive candidates in the potential fabrication of low power, supersensitive, and rapid response sensors and rectifiers.

The $p-n$ diodes were fabricated from $\mathrm{SnO}_{2}$ nanoribbons and P3HT nanofibers prepared via electrospinning. The sols used in the electrospinning process were prepared as follows: $2 \mathrm{ml}$ of commercially available tin(IV) isopropoxide, $10 \%$ $w / v$ in isopropanol/toluene (Alfa-Aesar) was thoroughly mixed with a $0.5 \mathrm{ml}$ of a 0.7 wt \% polyethylene oxide (MW 2000,000) (PEO) in $\mathrm{CHCl}_{3}$. In a separate vial, $26 \mathrm{mg}$ of regioregular $\mathrm{P} 3 \mathrm{HT}$ (Aldrich) and $1 \mathrm{mg}$ of $\mathrm{PEO}$ were dissolved in $3.0 \mathrm{~g}$ of $\mathrm{CHCl}_{3}$. First, the tin precursor was electrospun in air at $10 \mathrm{kV}$ and doped $\mathrm{Si}^{++}(0.01-0.05 \Omega-\mathrm{cm})$

${ }^{\text {a)} E l e c t r o n i c ~ m a i l: ~ n i c h o l a s . p i n t o @ u p r . e d u . ~}$ wafers with a $200 \mathrm{~nm}$ oxide layer were used to capture long (several $\mathrm{mm}$ ) ribbons of the precursor. ${ }^{8}$ The substrates were then heated in air at $700{ }^{\circ} \mathrm{C}$ for two hours to yield $\mathrm{SnO}_{2}$ nanoribbons that were seen to firmly adhere to the substrate. The P3HT sol was then electrospun in air at $5 \mathrm{kV}$ and individual P3HT nanofibers were captured on the same substrates as before. Several P3HT nanofibers were seen to intersect the $\mathrm{SnO}_{2}$ ribbons, each intersection resulting in a $p-n$ diode. For electrical characterization, one such junction was identified and electrical contact pads evaporated using an appropriate transmission electron microscope (TEM) grid as a shadow mask. Figure 1(a) shows an optical microscope image of the $\mathrm{P} 3 \mathrm{HT}$ nanofiber intersecting a $\mathrm{SnO}_{2}$ nanoribbon, together with portions of the gold contacts. Using an atomic force microscope, the ribbon and the fiber height were measured to be around $100 \mathrm{~nm}$. The P3HT nanofiber was about $2 \mu \mathrm{m}$ and the $\mathrm{SnO}_{2}$ ribbon was about $30 \mu \mathrm{m}$ wide. External electrical contacts were then made and the device currentvoltage $(I-V)$ characteristics were measured via a Keithley 6517A electrometer in a vacuum of $\sim 2 \times 10^{-2}$ Torr. The inset in Fig. 1(a) shows a schematic of the device and the external electrical circuit. Diodes were also fabricated by simply placing a $\mathrm{P} 3 \mathrm{HT} / \mathrm{CHCl}_{3}$ drop over the $\mathrm{SnO}_{2}$ nanoribbon as seen in Fig. 1(b) and electrically characterized.

Figure 2 shows the $I-V$ characteristic curves at $296 \mathrm{~K}$ of the device shown in Fig. 1(a) tested in air and in vacuum. Several diodes were tested and, in general, the $I-V$ character-

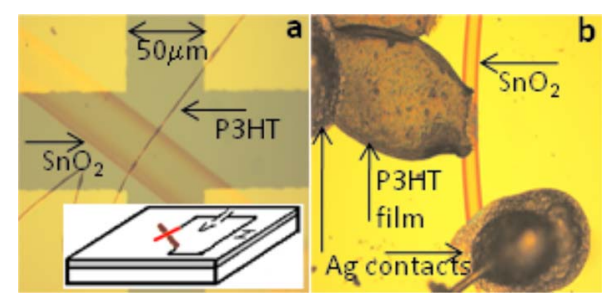

FIG. 1. (Color online) (a) Optical microscope image of a $\mathrm{SnO}_{2}$ ribbon crossed with a P3HT nanofiber. The inset is a schematic of the external electrical connections. (b) Optical microscope image of a $\mathrm{SnO}_{2}$ ribbon crossed with a P3HT cast film. The ribbon width was $\sim 60 \mu \mathrm{m}$ and the silver contacts are made directly to the active materials. 


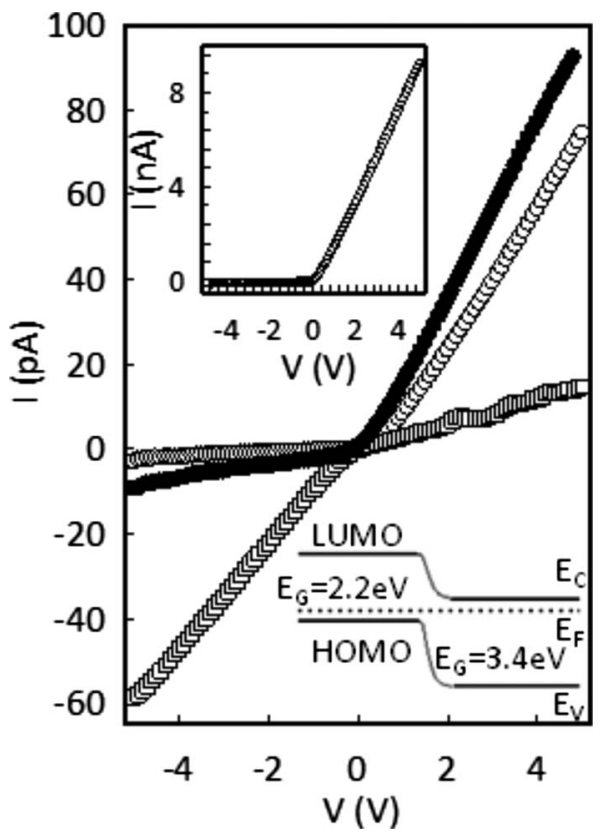

FIG. 2. Current-voltage $(I-V)$ characteristics for the device shown in Fig. 1(a) measured in air (-filled symbols) and in vacuum ( $\bigcirc$-empty symbols) when a positive bias was applied to the P3HT arm with the negative bias on the $\mathrm{SnO}_{2}$ arm and when the bias terminals were reversed $(\square)$. The inset above shows the $I-V$ characteristic curves for a device fabricated from a $\mathrm{SnO}_{2}$ ribbon crossed with a P3HT cast film shown in Fig. 1(b). The inset below shows the energy band diagram for the $p$ - $n$ diode.

istic curves were asymmetrical with a turn-on voltage in the range of $0.2-0.4 \mathrm{~V}$ and a much reduced reverse bias current. These devices exhibited rectifying behavior and the ratio of the forward to reverse current at a bias voltage of $\pm 1 \mathrm{~V}$ for this device was calculated to be $\sim 25$. The upper inset to Fig. 2 shows the $I-V$ curve for a diode fabricated with a drop cast film of P3HT (instead of the nanofiber) over a $\mathrm{SnO}_{2}$ ribbon. The measured currents are larger due to the increased film thickness and hence lower resistance of the film compared to the nanofiber. This diode also shows rectification with a turn-on voltage of $\sim 0.35 \mathrm{~V}$ and a rectification ratio of 70 . Prolonged device exposure to air (several days) reduces the rectification ratio.

In order to verify the $n$ - and the $p$-type doping of $\mathrm{SnO}_{2}$ and $\mathrm{P} 3 \mathrm{HT}$, respectively, electrospun ribbons and nanofibers of each were captured separately on doped $\mathrm{Si} / \mathrm{SiO}_{2}$ substrates and connected in a field effect transistor (FET) configuration. Figure 3 shows the device drain-source current $\left(I_{\mathrm{DS}}\right)$ as a function of drain-source voltage $\left(V_{\mathrm{DS}}\right)$ for different back gate biases $\left(V_{\mathrm{GS}}\right)$. The increase in $I_{\mathrm{DS}}$ for increasing positive $V_{\mathrm{GS}}$ for $\mathrm{SnO}_{2}$ and the increase in $I_{\mathrm{DS}}$ for increasing negative $V_{\mathrm{GS}}$ for $\mathrm{P} 3 \mathrm{HT}$ confirm the nature of the doping for each semiconductor. These observations further strengthen our claim that the fabricated $\mathrm{SnO}_{2} / \mathrm{P} 3 \mathrm{HT}$ crossed diodes are $p$ - $n$ rather than the Schottky type. In the linear regime of the $I_{\mathrm{DS}}-V_{\mathrm{DS}}$ curve, the device transconductance is $g_{m}$ $=\left(\partial I_{\mathrm{DS}} / \partial V_{\mathrm{GS}}\right)_{V_{\mathrm{DS}}=\mathrm{const}}$ and the carrier mobility is then determined from $\mu=g_{m} L / Z C_{i} V_{\mathrm{DS}}$, where $L$ and $Z$ are the channel length and width, respectively. $C_{i}$ is the capacitance per unit area of the $200 \mathrm{~nm}$ thick $\mathrm{SiO}_{2}$ layer $\left(=17.5 \mathrm{nF} / \mathrm{cm}^{2}\right)$. From Fig. 3, we estimate the charge mobility for the $\mathrm{SnO}_{2}$ FET $(L=110 \mu \mathrm{m}, Z=5 \mu \mathrm{m})$ to be $3 \times 10^{-3} \mathrm{~cm}^{2} / \mathrm{V} \mathrm{s}$ and for the P3HT FET ( $L=10 \mu \mathrm{m}, Z=2 \mu \mathrm{m})$ to be $2 \times 10^{-3} \mathrm{~cm}^{2} / \mathrm{V} \mathrm{s}$, lower than reported values. ${ }^{3,12,13}$ Since the electrospinning

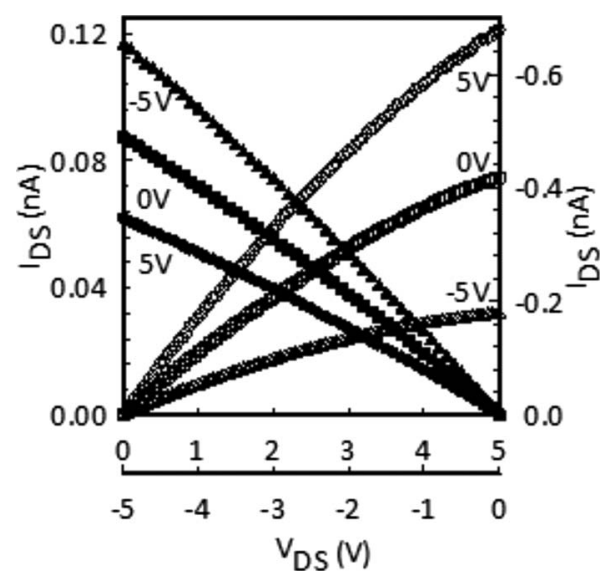

FIG. 3. (a) Drain source current $\left(I_{\mathrm{DS}}\right)$ vs drain source voltage $\left(V_{\mathrm{DS}}\right)$ curves for a single electrospun $\mathrm{SnO}_{2}$ ribbon (open symbols-first quadrant axes) when placed in a FET configuration for different gate bias voltages $(-5,0$, and $5 \mathrm{~V})$. (b) Drain source current $\left(I_{\mathrm{DS}}\right)$ vs drain source voltage $\left(V_{\mathrm{DS}}\right)$ curves for a single electrospun P3HT nanofiber (filled symbols-third quadrant axes) when placed in a FET configuration for different gate bias voltages $(-5,0$, and $5 \mathrm{~V})$.

technique likely produces ribbons and fibers that are amorphous due to the rapid evaporation of the solvent, it is not surprising that charge mobility in these devices is small. Treating the $\mathrm{SiO}_{2}$ with a monolayer of octyltrichlorosilane is expected to increase charge mobility.

The band gaps in $\mathrm{P} 3 \mathrm{HT}$ and $\mathrm{SnO}_{2}$ are approximately 2.2 and $3.4 \mathrm{eV}$, respectively, and when used in a FET configuration, show that they make Ohmic contacts with $\mathrm{Au}(5.1 \mathrm{eV})$ electrodes. The essential features of the asymmetric nonlinear $I-V$ curves in Fig. 2 can thus be understood qualitatively based on the energy band diagram for a planar structure in bulk $p$ - $n$ junctions. ${ }^{14}$ Diffusion of charge carriers (polarons in $\mathrm{P} 3 \mathrm{HT}$ and electrons in $\mathrm{SnO}_{2}$ ) across the junction sets up an electric field of opposite polarity that prevents further diffusion and creates a space charge region depleted of all mobile charge across the interface. Band bending through the space charge region helps establish a constant Fermi level in thermal equilibrium resulting in a built-in potential barrier to the flow of electrons from the conduction band of $\mathrm{SnO}_{2}$ moving into the lowest unoccupied molecular orbital level of P3HT, as shown in the lower inset to Fig. 2. Applying a positive potential to the $n$-region with respect to the $p$-region lowers the Fermi energy in the $n$-region increasing the barrier height, preventing charge diffusion, and hence limiting current flow (reverse bias). Applying a positive potential to the $p$-region with respect to the $n$-region lowers the Fermi energy in the $p$-region, decreasing the barrier height in the $p$-region and allowing for charge diffusion across the junction, which constitutes a current (forward bias) that increases exponentially under bias voltage as governed by the FermiDirac occupancy function. Under such conditions, in an ideal diode, the current-voltage curve can be described by the equation ${ }^{14} I=I_{S}\left[\exp \left(e V / n k_{B} T\right)-1\right]$, where $I_{S}$ is the reverse bias saturation current, $e$ is the electronic charge, and $n$ represents the ideality factor; $k_{B}$ and $T$ represent the Boltzmann factor and temperature, respectively. Figure 4 shows the diode characteristics near the turn-on region and the inset shows the semilogarithmic plot of the diode current versus applied voltage under forward bias conditions. Extrapolating the linear portion of the semilog plot to zero bias yields a 


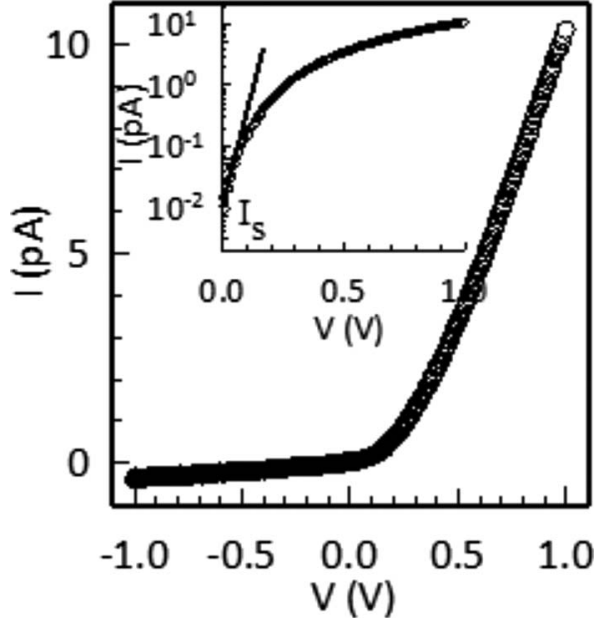

FIG. 4. Expanded plot at low bias voltage of the $I-V$ curve shown in Fig. 2. Inset: semilog plot of the forward bias current as a function of forward bias voltage.

reverse bias saturation current of $0.022 \mathrm{pA}$ and the diode ideality factor was calculated to be $n \approx 2.2$.

Being transparent, $\mathrm{SnO}_{2}$ is insensitive to visible light, but its conductivity is enhanced in the presence of UV light $(\lambda=365 \mathrm{~nm})$. This is most likely due to the removal of adsorbed oxygen species releasing the surface bound electrons that contribute to the observed current and due to photoexcitation of electrons from the valence to the conduction band, ${ }^{3,4}$ which motivated us to test the diode as a light sensor. Figure 5 shows these results in an as prepared device measured in vacuum, before, during, and after exposure to UV light. The on state current increases in the presence of

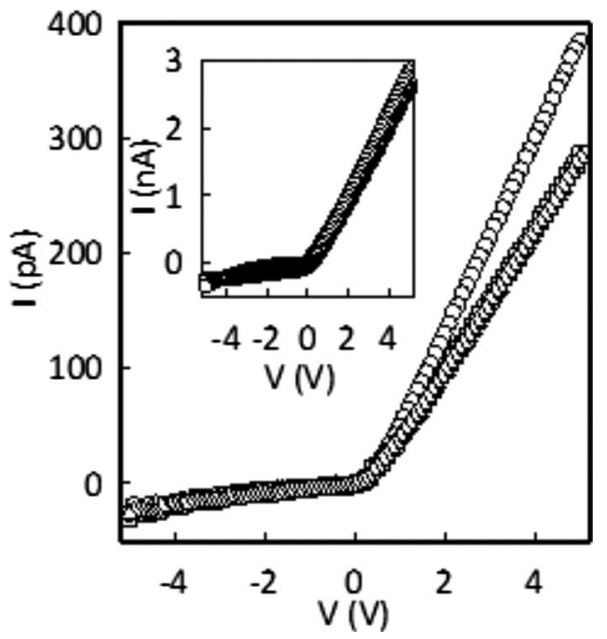

FIG. 5. Current-voltage $(I-V)$ characteristic curves for the $p-n$ diode when used as a UV light sensor: $(\square)$ in the absence of UV, $(\bigcirc)$ in the presence of $(\mathrm{UV})$, and $(\triangle)$ after the UV light source was switched off. Inset: $I-V$ characteristic curves for the device fabricated from a $\mathrm{SnO}_{2}$ ribbon crossed with a P3HT drop cast film: $(\square)$ in the absence of UV and $(O)$ in the presence of UV.
UV and the device returns to its original state after UV exposure. The inset in Fig. 5 shows the same measurements on a diode made from a thin $\mathrm{P} 3 \mathrm{HT}$ film cast over the $\mathrm{SnO}_{2}$ ribbon. While the response is similar, the relative changes are larger in the "nanofiber" diode due to the increased surface to volume ratio of the fiber over the film and confirms the superior nature of such diodes when used as sensors.

In summary, we present a facile and inexpensive method of fabricating $p$ - $n$ diodes in air and within seconds using the electrospinning technique. The diodes show clear evidence of rectification in air or in vacuum, under ambient illumination or in the dark, with a rectification ratio of $\sim 25$. The device was analyzed using the diode equation and yielded an ideality parameter of $\sim 2.2$ and a turn-on voltage in the range of $0.2-0.4 \mathrm{~V}$. These low turn-on voltages are beneficial for polymer based electronics that currently operate under high biases. Device parameters could be improved by using crystalline materials at the expense however of compromising the simple fabrication technique outlined here. UV light exposure increases the diode on state current, while light removal resets the device. Diodes were also fabricated by drop casting films of P3HT over individual $\mathrm{SnO}_{2}$ ribbons, but were relatively less sensitive to UV light. The high surface to volume ratio of the nanofiber, low turn-on voltages, and response to UV light, make this device attractive for use as low power consumption diodes and reusable sensors.

This work was supported in part by NSF under Grant Nos. RUI-0703544 and PREM-0353730 and by the DoD under Grant No. W911NF-06-1-0519. R.A. acknowledges support from the NSF-CAREER Grant No. ECCS-0644737 and the Science and Engineering grant from Dupont.

${ }^{1}$ C. K. Chiang, C. R. Fincher, Jr., Y. W. Park, A. J. Heeger, H. Shirakawa, E. J. Louis, S. C. Gau, and A. G. MacDiarmid, Phys. Rev. Lett. 39, 1098 (1977).

${ }^{2}$ G. Eranna, B. C. Joshi, D. P. Runthala, and R. P. Gupta, Crit. Rev. Solid State Mater. Sci. 29, 111 (2004).

${ }^{3}$ Z. Liu, D. Zhang, S. Han, C. Li, T. Tang, W. Jin, X. Liu, B. Lei, and C. Zhou, Adv. Mater. (Weinheim, Ger.) 15, 1754 (2003).

${ }^{4}$ S. Mathur, S. Barth, H. Shen, J.C. Pyun and U. Werner, Small 1, 713 (2005).

${ }^{5}$ D. P. Halliday, J. W. Gray, P. N. Adams, and A. P. Monkman, Synth. Met. 102, 877 (1999).

${ }^{6}$ O. Inganäs, T. Skotheim, and I. Lundström, J. Appl. Phys. 54, 3636 (1983).

${ }^{7}$ M. C. Lonergan, Science 278, 2103 (1997).

${ }^{8}$ N. J. Pinto, R. Gonzalez, A. T. Johnson, Jr., and A. G. MacDiarmid, Appl. Phys. Lett. 89, 033505 (2006).

${ }^{9}$ F. Kong, Y. Wang, J. Zhang, H. Xia, B. Zhu, Y. Wang, S. Wang, and S. Wu, Mater. Sci. Eng., B 150, 6 (2008).

${ }^{10}$ X. Ai, N. Anderson, J. Guo, J. Kowalik, L. M. Tolbert, and T. Lian, J. Phys. Chem. 110, 25496 (2006)

${ }^{11}$ M. K. Ram, O. Yavuz, and M. Aldissi, Synth. Met. 151, 77 (2005).

${ }^{12}$ H. Liu, C. H. Reccius, and H. G. Craighead, Appl. Phys. Lett. 87, 253106 (2005).

${ }^{13}$ A. Babel, D. Li, Y. Xia, and S. A. Jenekhe, Macromolecules 38, 4705 (2005).

${ }^{14}$ D. A. Neamen, Semiconductor Physics and Devices, 3rd ed. (McGrawHill, New York, 2003), Chaps. 7 and 8. 